\title{
Obstetric and perinatal health outcomes after pertussis vaccination during pregnancy in Ontario, Canada: a retrospective cohort study
}

\author{
Romina Fakhraei MSc, Natasha Crowcroft MD(Cantab) PhD, Shelly Bolotin PhD, Ewa Sucha MSc, \\ Steven Hawken PhD, Kumanan Wilson MD MSc, Laura Gaudet MD MSc, Gayatri Amirthalingam MD, \\ Anne Biringer MD, Jocelynn Cook PhD, Vinita Dubey MD MPH, Scott A. Halperin MD, \\ Frances Jamieson MD, Jeffrey C. Kwong MD MSc, Manish Sadarangani MD PhD, \\ Mark C. Walker MD MSc, Meghan Laverty MSc, Deshayne B. Fell PhD
}

\section{Abstract}

Background: In February 2018, Canada's National Advisory Committee on Immunization recommended maternal vaccination with tetanus-diphtheria-acellular pertussis (Tdap) vaccine during pregnancy to prevent severe pertussis infection in young infants. This study assessed the relation between maternal Tdap vaccination and obstetric and perinatal outcomes in Ontario.

Methods: We performed a population-based cohort study of all births from April 2012 to March 2017 using multiple linked health administrative databases. We used Cox regression with a time-dependent exposure variable to estimate adjusted hazard ratios (HRs) for preterm birth (<37 wk), very preterm birth (<32 wk) and stillbirth. We assessed remaining outcomes (gestational hypertension, chorioamnionitis, postpartum hemorrhage, severe postpartum hemorrhage, being small for gestational age, neonatal intensive care unit stay $>24 \mathrm{~h}$, composite neonatal morbidity) using log-binomial regression to generate adjusted risk ratios (RRs). We adjusted estimates for potential confounding using propensity score weighting.

Results: Of 615213 infants (live births and stillbirths), 11519 were exposed to Tdap vaccination in utero. There was no increased risk for preterm birth (adjusted HR 0.98, 95\% confidence interval [Cl] 0.91-1.06), very preterm birth (adjusted $\mathrm{HR} 1.10,95 \% \mathrm{Cl} 0.86-$ 1.41), stillbirth (adjusted HR 1.15, 95\% Cl 0.82-1.60) or being small for gestational age (adjusted RR 0.96, 95\% Cl 0.90-1.02). The risks of a neonatal intensive care unit stay exceeding 24 hours (adjusted RR $0.82,95 \% \mathrm{Cl} 0.76-0.88$ ) and neonatal morbidity (adjusted RR $0.81,95 \% \mathrm{Cl} 0.75-0.87$ ) were decreased. There was no association with chorioamnionitis (adjusted RR 1.17, 95\% Cl 0.99-1.39), postpartum hemorrhage (adjusted RR 1.01, 95\% Cl 0.91-1.13) or severe postpartum hemorrhage (adjusted RR 0.79, 95\% Cl 0.55-1.13), but we observed a reduced risk of gestational hypertension (adjusted RR 0.87, 95\% Cl 0.78-0.96).

Interpretation: Our results complement evidence that maternal Tdap vaccination is not associated with adverse outcomes in mothers or infants. Ongoing evaluation in Canada is needed as maternal Tdap vaccination coverage increases in coming years.

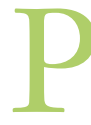

ertussis, a vaccine-preventable disease, remains a public health challenge in many countries, including Canada. ${ }^{1-5}$ Infants less than 1 year of age who have not completed their primary vaccine series are at highest risk for pertussis-related morbidity and mortality. ${ }^{6,7}$ Large outbreaks with increased incidence and deaths in young infants $^{8-11}$ prompted the United States in 2011 and United Kingdom in 2012 to recommend vaccination of pregnant women with tetanus-diphtheria-acellular pertussis (Tdap) vaccine to protect newborns. ${ }^{12,13}$ Canada's National Advisory Committee on Immunization adopted a similar recommendation in $2018 ;{ }^{14}$ however, some maternity care providers were already vaccinating pregnant women against pertussis during the preceding 5 years, likely based on international programs ${ }^{12,13}$ and earlier Canadian recommendations. ${ }^{15,16}$
Competing interests: Scott Halperin has received research grants and contracts from and has served on ad hoc advisory boards for GlaxoSmithKline and Sanofi Pasteur, manufacturers of tetanus-diphtheriaacellular pertussis vaccines, unrelated to this study. Manish Sadarangani is supported by salary awards from the BC Children's Hospital Foundation, the Canadian Child Health Clinician Scientist Program and the Michael Smith Foundation for Health Research. He has been an investigator on projects funded by Pfizer, Merck, Seqirus, VBI Vaccines, GlaxoSmithKline and Sanofi Pasteur; all funds have been paid to his institute, and he has not received any personal payments. Kumanan Wilson is married to an employee of CMAJ, who was not involved with the editorial decisionmaking process for this article. No other competing interests were declared.

This article has been peer reviewed.

Correspondence to: Deshayne Fell, dfell@cheo.on.ca

CMAJ Open 2021. DOI:10.9778/cmajo.20200239 
The literature on the safety of maternal pertussis vaccination ${ }^{17-30}$ focuses predominantly on the US and UK, and only a limited number of smaller studies have evaluated stillbirth. ${ }^{17,19,21,23,31-33}$ Moreover, inconsistent findings from earlier studies concerning the risk of chorioamnionitis ${ }^{18,27,30}$ and postpartum hemorrhage ${ }^{30}$ warrant further investigation in other populations. To provide baseline evidence on maternal Tdap vaccination in Canada, we assessed whether receipt of Tdap vaccine during pregnancy was associated with adverse obstetric or perinatal outcomes in Ontario.

\section{Methods}

\section{Study design, data sources and study population}

We conducted a population-based retrospective cohort study of all hospital live births and stillbirths in Ontario between Apr. 1, 2012, and Mar. 3, 2017. We used the MOMBABY database, which contains linked maternal-newborn hospital records, to assemble the cohort and provide additional information (e.g., gestational age, birth weight). About 99\% of maternal-newborn records for in-hospital deliveries in Ontario have been successfully linked in this database. ${ }^{34} \mathrm{We}$ linked 5 other health administrative databases: the Registered Persons Database, which provides information on neighbourhood income, region of residence and health care eligibility; the Canadian Institute for Health Information Discharge Abstract Database, which captures hospital admissions (medical diagnoses and procedures); the Ontario Health Insurance Plan database, which contains physician billing claims; the Permanent Resident Database (Immigration, Refugees and Citizenship Canada), which includes maternal country of birth; and the Ontario Marginalization Index, which uses census data to quantify the level of marginalization. Data sources are described in Appendix 1, Supplemental Table S1 (available at www.cmajopen.ca/content/9/2/E349/suppl/DC1).

Data sets were linked by means of unique encoded identifiers and analyzed at ICES. We obtained diagnostic and procedural codes from the enhanced Canadian version of the International Statistical Classification of Diseases and Related Health Problems, 10th Revision (ICD-10-CA) and the Canadian Classification of Health Interventions, respectively.

For multifetal pregnancies, only the first-born infant was included. Excluded were mothers who were not Ontario residents, were younger than 12 years or older than 50 years, or did not have continuous Ontario Health Insurance Plan enrolment during pregnancy, and records with administrative issues (e.g., invalid identifiers, duplicate records). We applied an algorithm ${ }^{35,36}$ to exclude live births with implausible birth weight and gestational age combinations, based on a Canadian reference standard. ${ }^{37}$ Finally, records missing covariate information were excluded.

\section{Exposure and outcome measurement}

We identified Tdap vaccination during pregnancy using billing code G847 in the Ontario Health Insurance Plan database. We classified Tdap vaccination as having occurred during pregnancy if it was administered between 14 days after the last menstrual period (estimated by subtracting gestational age from date of birth) to 1 day before delivery. We categorized gestational age at Tdap vaccine receipt as less than 20 weeks, 20-26 weeks, 27-32 weeks (recommended timing ${ }^{14}$ ) and more than 32 weeks.

Obstetric and perinatal outcomes were chosen based on prior vaccine research, importance to perinatal health and international recommendations on vaccination. ${ }^{18,27,30,38}$ Obstetric outcomes of interest were gestational hypertension, chorioamnionitis, postpartum hemorrhage and severe postpartum hemorrhage (postpartum hemorrhage combined with hysterectomy, blood transfusion or other procedures to control bleeding). We identified these outcomes using ICD10-CA codes from any maternal hospitalization record during pregnancy, including delivery.

We examined 6 perinatal outcomes: stillbirth (at $\geq 20 \mathrm{wk}$ of gestation), preterm birth $(<37 \mathrm{wk})$, very preterm birth $(<32 \mathrm{wk})$, small for gestational age $(<10$ th percentile for gestational age- and sex-specific birth weight ${ }^{37}$ ), neonatal intensive care unit stay longer than 24 hours, and an adaptation ${ }^{39}$ of a composite indicator for neonatal morbidity ${ }^{40}$ that flags infants with 1 or more of 15 neonatal diagnoses or 7 procedures within 28 days after birth (codes provided in Appendix 1, Supplemental Tables S2 and S3). The denominator for analyses of stillbirth, preterm birth and obstetric outcomes included all live births and stillbirths (cohort 1); we restricted the cohort to live births for analyses of the remaining perinatal outcomes (cohort 2).

\section{Statistical analysis}

We used frequencies to describe the distribution of baseline categoric variables and standardized differences to compare distributions between vaccine-exposed and -unexposed mothers, with an absolute value of 0.10 or greater considered indicative of imbalance. ${ }^{41,42}$ To assess preterm birth and stillbirth, we used an extension of Cox regression, with a timedependent exposure variable and gestational age in days as the time scale, to generate hazard ratios (HRs) and 95\% confidence intervals (CIs). Follow-up for these time-to-event outcomes commenced 14 days after the last menstrual period; those who did not experience the event were censored at 37 weeks (preterm birth), 32 weeks (very preterm birth) ${ }^{43}$ or birth (stillbirth). For the remaining outcomes, we used logbinomial regression to compute risk ratios (RRs) and $95 \%$ CIs. We used robust sandwich estimators (Cox models) and general estimating equations with independent correlation structure (log-binomial models) to account for multiple deliveries to 1 mother.

To generate results adjusted for confounding, we used propensity score methods. We generated a propensity score representing the probability of Tdap vaccine receipt for each mother, using a logistic regression model including all baseline covariates as well as adequacy of prenatal care ${ }^{44}$ (Appendix 1, Supplemental Tables S2 and S4, and section on coding algorithm for Revised-Graduated Prenatal Care Utilization Index). We transformed the propensity scores into stabilized inverse probability of treatment weights, which were 
incorporated in all regression models to generate adjusted estimates (Appendix 1, section on propensity score adjustment). ${ }^{45}$ All analyses were conducted with SAS Enterprise Guide 7.1 (SAS Institute).

We assessed the impact of our analytical decisions through several sensitivity analyses. First, ascertainment of Tdap vaccination has been found to have improved sensitivity when the pertussis-specific vaccine code, introduced in 2011, is used in combination with general vaccine codes (G538, G539) in years proximal to when vaccine-specific codes were introduced. ${ }^{46}$ Our sensitivity analysis also included general vaccine codes billed during pregnancy (but not between Oct. 1 and Jan. 31, as these may have been influenza vaccinations) in an expanded exposure definition additionally capturing possible Tdap vaccination (Appendix 1, Supplemental Table S2). Second, to account for potential differences in maternal health care seeking or access, we also adjusted for number of outpatient visits and nonobstetric hospital admissions within 6 months and 2 years before the index pregnancy, respectively (Appendix 1, Supplemental Table S2). Third, using the array approach, ${ }^{47}$ we estimated the impact of an unmeasured confounder (Appendix 1, section on bias analyses).

\section{Ethics approval}

Research ethics approval was obtained from the Children's Hospital of Eastern Ontario Research Ethics Board (protocol 18/10PE), the Ottawa Health Science Network Research Ethics Board (protocol 20180432-01H) and the ICES Privacy Office (protocol 20180901166 000).

\section{Results}

After exclusions, there were 615213 live births and stillbirths; 11519 infants (1.9\%) were born to mothers who had received Tdap vaccine during pregnancy (Figure 1). In the unweighted population, compared to unvaccinated mothers, Tdapvaccinated mothers were more likely to be nulliparous, have conceived in later study years, reside in the eastern region of the province, and reside in neighbourhoods with the highest income level and least material deprivation. After weighting, the distributions of all baseline characteristics were similar by exposure status, with no standardized differences exceeding 0.1 (Table 1; Appendix 1, Supplemental Figure S1).

\section{Obstetric outcomes}

Of the 11519 Tdap-vaccinated mothers, 2631 (22.8\%) were vaccinated before 20 weeks' gestation, $1130(9.8 \%)$ at 20-26 weeks' gestation, 4774 (41.1\%) at 27-32 weeks' gestation, and 2984 (25.9\%) after 32 weeks' gestation. Although the cumulative incidence of chorioamnionitis was slightly higher among vaccinated than unvaccinated mothers $(1.2 \% \mathrm{v}$. $1.0 \%)$, there was no association after propensity score weighting (adjusted RR 1.17, 95\% CI 0.99-1.39) (Table 2). Receipt of Tdap vaccine during pregnancy was not significantly associated with postpartum hemorrhage (adjusted RR $1.01,95 \%$ CI $0.91-1.13$ ) or severe postpartum hemorrhage (adjusted RR 0.79, 95\% CI 0.55-1.13), but we observed an inverse association between Tdap vaccination and gestational hypertension, even after adjustment (adjusted RR 0.87, 95\% CI 0.78-0.96).

\section{Perinatal outcomes}

The cumulative incidence of preterm birth was similar in the 2 exposure groups (Tdap-vaccinated $6.3 \%$, unvaccinated $7.4 \%)$, and there was no increased risk associated with vaccination (adjusted HR 0.98, 95\% CI 0.91-1.06) (Table 2). We did not observe an association between maternal Tdap vaccination and stillbirth (adjusted HR 1.15, 95\% CI 0.82-1.60) or being small for gestational age (adjusted RR 0.96, 95\% CI 0.90-1.02). Even after accounting for confounding, we found reduced risks of a neonatal intensive care unit stay longer than 24 hours (adjusted RR 0.82, 95\% CI 0.76-0.88) and composite neonatal morbidity (adjusted RR 0.81, 95\% CI 0.75-0.87) with vaccination.

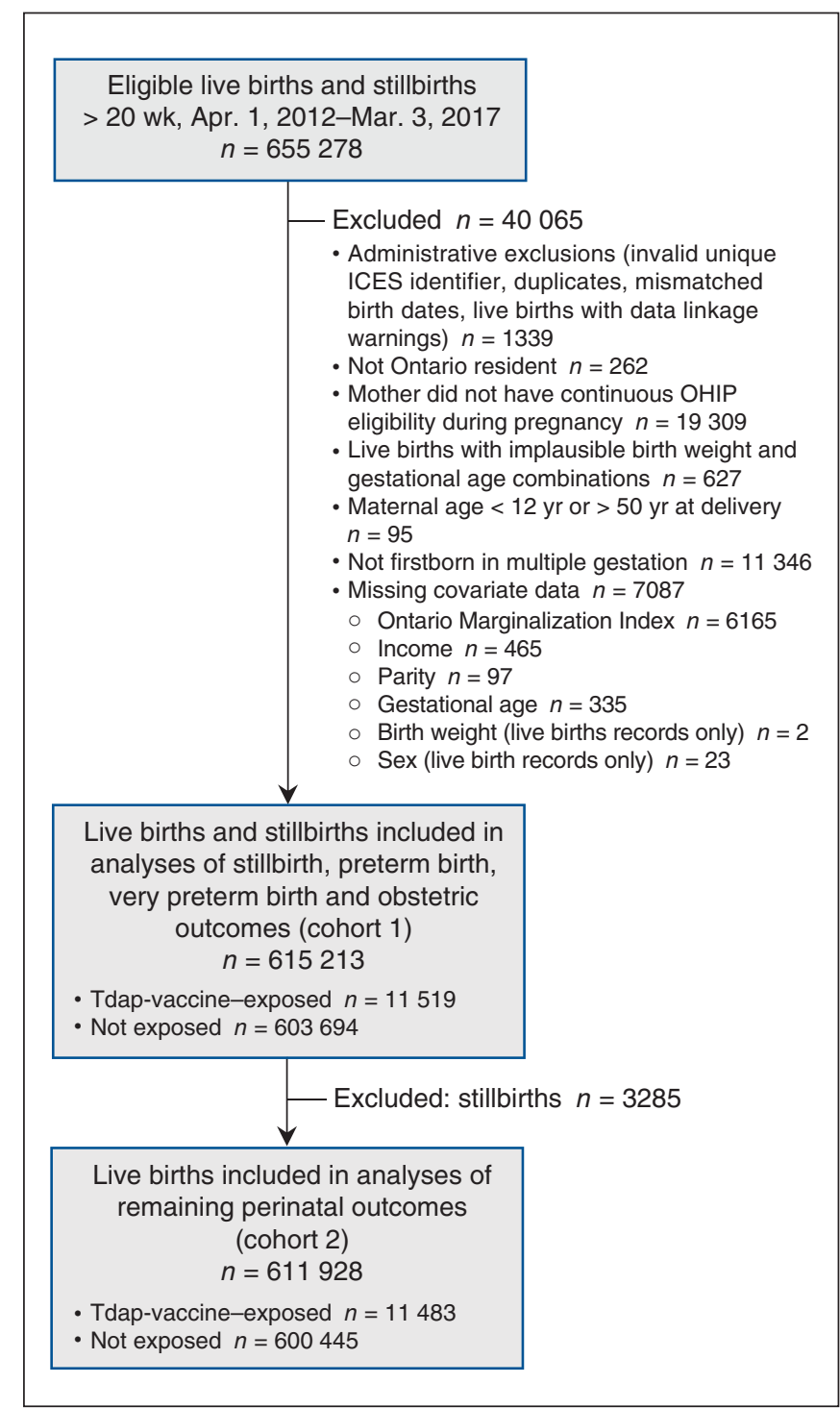

Figure 1: Flow diagram showing creation of study cohorts. Note: $\mathrm{OHIP}=$ Ontario Health Insurance Plan, Tdap = tetanus-diphtheriaacellular pertussis. 
Table 1 (part 1 of 2): Baseline and birth characteristics of hospital live births and stillbirths in Ontario between Apr. 1, 2012, and Mar. 3, 2017, before and after propensity score weighting, by tetanus-diphtheria-acellular pertussis vaccination status (cohort 1)

\begin{tabular}{|c|c|c|c|c|c|c|c|}
\hline \multirow[b]{2}{*}{ Characteristic } & \multirow{2}{*}{$\begin{array}{c}\text { Total population, } \\
\text { no. (\%) of } \\
\text { mothers } \\
n=615213\end{array}$} & \multicolumn{2}{|c|}{$\begin{array}{l}\text { Unweighted cohort, no. (\%) } \\
\text { of mothers }\end{array}$} & \multirow[b]{2}{*}{$\begin{array}{c}\text { Standardized } \\
\text { difference } \dagger\end{array}$} & \multicolumn{2}{|c|}{$\begin{array}{l}\text { IPTW-weighted cohort, }{ }^{*} \\
\% \text { of mothers }\end{array}$} & \multirow[b]{2}{*}{$\begin{array}{l}\text { Standardizec } \\
\text { difference }\end{array}$} \\
\hline & & $\begin{array}{l}\text { Vaccinated } \\
n=11519\end{array}$ & $\begin{array}{l}\text { Unvaccinated } \\
n=603694\end{array}$ & & Vaccinated & Unvaccinated & \\
\hline \multicolumn{8}{|l|}{ Maternal age, yr } \\
\hline$<20$ & $14249(2.3)$ & $182(1.6)$ & $14067(2.3)$ & 0.05 & 3.0 & 2.3 & 0.04 \\
\hline $20-24$ & $66102(10.7)$ & $788(6.8)$ & 65314 (10.8) & 0.14 & 10.2 & 10.9 & 0.02 \\
\hline $25-29$ & $165891(27.0)$ & $3272(28.4)$ & $162619(26.9)$ & 0.03 & 28.1 & 26.9 & 0.03 \\
\hline $30-34$ & $225692(36.7)$ & 4536 (39.4) & $221156(36.6)$ & 0.06 & 35.4 & 36.6 & 0.02 \\
\hline$\geq 35$ & 143279 (23.3) & $2741(23.8)$ & 140538 (23.3) & 0.01 & 23.3 & 23.3 & 0.00 \\
\hline \multicolumn{8}{|l|}{ Year of conception } \\
\hline $2011 / 12$ & 95499 (15.5) & 385 (3.3) & $95114(15.8)$ & 0.43 & 19.0 & 16.0 & 0.08 \\
\hline $2012 / 13$ & $124714(20.3)$ & $1323(11.5)$ & $123391(20.4)$ & 0.25 & 20.4 & 20.6 & 0.00 \\
\hline $2013 / 14$ & $124990(20.3)$ & $2043(17.7)$ & $122947(20.4)$ & 0.07 & 19.1 & 20.4 & 0.03 \\
\hline $2014 / 15$ & $124163(20.2)$ & $2426(21.1)$ & $121737(20.2)$ & 0.02 & 19.3 & 20.1 & 0.02 \\
\hline $2015 / 16$ & $124232(20.2)$ & 4478 (38.9) & $119754(19.8)$ & 0.43 & 18.8 & 19.5 & 0.02 \\
\hline $2016 / 17$ & 21615 (3.5) & $864(7.5)$ & $20751(3.4)$ & 0.18 & 3.4 & 3.4 & 0.00 \\
\hline \multicolumn{8}{|l|}{ Parity } \\
\hline 0 & 272399 (44.3) & $6027(52.3)$ & $266372(44.1)$ & 0.16 & 43.0 & 44.0 & 0.02 \\
\hline$\geq 1$ & 342814 (55.7) & $5492(47.7)$ & $337322(55.9)$ & 0.16 & 57.0 & 56.0 & 0.02 \\
\hline Multiple birth & $11734(1.9)$ & $155(1.3)$ & $11579(1.9)$ & 0.05 & 2.0 & 1.9 & 0.00 \\
\hline \multicolumn{8}{|l|}{ Baby’s sex $\ddagger$} \\
\hline Female & $298032(48.4)$ & $5579(48.6)$ & $292453(48.7)$ & 0.00 & 48.6 & 48.4 & 0.00 \\
\hline Male & $313896(51.0)$ & $5904(51.4)$ & $307992(51.3)$ & 0.00 & 51.4 & 51.6 & 0.00 \\
\hline \multicolumn{8}{|c|}{ Preexisting maternal medical condition§ } \\
\hline Asthma & $1501(0.2)$ & $25(0.2)$ & $1476(0.2)$ & 0.01 & 0.3 & 0.3 & 0.01 \\
\hline $\begin{array}{l}\text { Chronic } \\
\text { hypertension }\end{array}$ & $2358(0.4)$ & $39(0.3)$ & $2319(0.4)$ & 0.01 & 0.4 & 0.4 & 0.01 \\
\hline Diabetes & $4846(0.8)$ & $55(0.5)$ & $4791(0.8)$ & 0.04 & 0.9 & 0.8 & 0.01 \\
\hline Heart disease & $2971(0.5)$ & $50(0.4)$ & $2921(0.5)$ & 0.01 & 0.5 & 0.5 & 0.01 \\
\hline Thyroid disease & $7135(1.2)$ & $199(1.7)$ & $6936(1.1)$ & 0.05 & 1.2 & 1.1 & 0.00 \\
\hline $\begin{array}{l}\text { Drug dependence or } \\
\text { tobacco use }\end{array}$ & $3326(0.5)$ & $37(0.3)$ & $3289(0.5)$ & 0.03 & 0.5 & 0.6 & 0.01 \\
\hline $\begin{array}{l}\text { Maternal obesity (body } \\
\text { mass index }>30.0 \text { ) }\end{array}$ & $3436(0.6)$ & $47(0.4)$ & $3389(0.6)$ & 0.02 & 0.5 & 0.6 & 0.01 \\
\hline \multicolumn{8}{|c|}{ Neighbourhood median family income quintile } \\
\hline 1 (lowest) & $131512(21.4)$ & $2240(19.4)$ & $129272(21.4)$ & 0.05 & 22.7 & 21.5 & 0.03 \\
\hline 2 & $123738(20.1)$ & $2466(21.4)$ & $121272(20.1)$ & 0.03 & 18.6 & 20.1 & 0.04 \\
\hline 3 & $127176(20.7)$ & 2215 (19.2) & $124961(20.7)$ & 0.04 & 21.2 & 20.7 & 0.01 \\
\hline 4 & $129987(21.1)$ & $2372(20.6)$ & $127615(21.1)$ & 0.01 & 21.7 & 21.1 & 0.01 \\
\hline 5 (highest) & $102800(16.7)$ & 2226 (19.3) & $100574(16.7)$ & 0.07 & 16.0 & 16.6 & 0.02 \\
\hline Rural residence & $57263(9.3)$ & $968(8.4)$ & $56295(9.3)$ & 0.03 & 9.4 & 9.3 & 0.00 \\
\hline \multicolumn{8}{|l|}{ LHIN group } \\
\hline Central & 204512 (33.2) & 3730 (32.4) & 200782 (33.3) & 0.02 & 33.2 & 33.3 & 0.00 \\
\hline East & $148737(24.2)$ & 4410 (38.3) & $144327(23.9)$ & 0.31 & 21.1 & 23.6 & 0.06 \\
\hline North & $32305(5.3)$ & $401(3.5)$ & 31904 (5.3) & 0.09 & 3.8 & 5.3 & 0.07 \\
\hline Toronto & $57397(9.3)$ & $1390(12.1)$ & 56007 (9.3) & 0.09 & 9.9 & 9.2 & 0.02 \\
\hline West & $172262(28.0)$ & $1588(13.8)$ & $170674(28.3)$ & 0.36 & 32.0 & 28.5 & 0.08 \\
\hline
\end{tabular}


Table 1 (part 2 of 2): Baseline and birth characteristics of all hospital live births and stillbirths in Ontario between Apr. 1, 2012, and Mar. 3, 2017, before and after propensity score weighting, by tetanus-diphtheria-acellular pertussis vaccination status (cohort 1)

\begin{tabular}{|c|c|c|c|c|c|c|c|}
\hline \multirow[b]{2}{*}{ Characteristic } & \multirow{2}{*}{$\begin{array}{l}\text { Total population, } \\
\text { no. }(\%) \text { of } \\
\text { mothers } \\
n=615213\end{array}$} & \multicolumn{2}{|c|}{$\begin{array}{l}\text { Unweighted cohort, no. (\%) } \\
\text { of mothers }\end{array}$} & \multirow[b]{2}{*}{$\begin{array}{l}\text { Standardized } \\
\text { difference }\end{array}$} & \multicolumn{2}{|c|}{$\begin{array}{l}\text { IPTW-weighted cohort, }{ }^{*} \\
\% \text { of mothers }\end{array}$} & \multirow[b]{2}{*}{$\begin{array}{l}\text { Standardized } \\
\text { difference† }\end{array}$} \\
\hline & & $\begin{array}{l}\text { Vaccinated } \\
n=11519\end{array}$ & $\begin{array}{l}\text { Unvaccinated } \\
n=603694\end{array}$ & & Vaccinated & Unvaccinated & \\
\hline \multicolumn{8}{|c|}{ Maternal world region of origin } \\
\hline North America & $473002(76.9)$ & $8706(75.6)$ & $464296(76.9)$ & 0.03 & 77.4 & 77.0 & 0.01 \\
\hline Asia & $89566(14.6)$ & $2198(19.1)$ & $87368(14.5)$ & 0.12 & 14.1 & 14.4 & 0.01 \\
\hline Europe & $12876(2.1)$ & $164(1.4)$ & $12712(2.1)$ & 0.05 & 2.1 & 2.1 & 0.00 \\
\hline Africa & $12467(2.0)$ & $160(1.4)$ & $12307(2.0)$ & 0.05 & 2.3 & 2.0 & 0.02 \\
\hline Caribbean & $8689(1.4)$ & $78(0.7)$ & $8611(1.4)$ & 0.07 & 1.3 & 1.4 & 0.01 \\
\hline $\begin{array}{l}\text { Yugoslavia/Soviet } \\
\text { Unionף }\end{array}$ & $8172(1.3)$ & $93(0.8)$ & $8079(1.3)$ & 0.05 & 1.3 & 1.4 & 0.00 \\
\hline South America & $6865(1.1)$ & $80(0.7)$ & $6785(1.1)$ & 0.05 & 1.0 & 1.1 & 0.02 \\
\hline Central America & $3270(0.5)$ & $28(0.2)$ & $3242(0.5)$ & 0.05 & 0.5 & 0.5 & 0.00 \\
\hline Oceania & $306(0.0)$ & $12(0.1)$ & $294(0.0)$ & 0.02 & 0.0 & 0.05 & 0.01 \\
\hline \multicolumn{8}{|c|}{ Ontario Marginalization Index dimension, quintile ${ }^{\star \star}$} \\
\hline \multicolumn{8}{|c|}{ Residential instability } \\
\hline 1 & 137422 (22.3) & 2351 (20.4) & 135071 (22.4) & 0.05 & 19.5 & 22.4 & 0.07 \\
\hline 2 & $114789(18.7)$ & $2230(19.4)$ & $112559(18.6)$ & 0.02 & 18.9 & 18.6 & 0.01 \\
\hline 3 & $110776(18.0)$ & $2070(18.0)$ & $108706(18.0)$ & 0.00 & 17.5 & 18.0 & 0.01 \\
\hline 4 & $115280(18.7)$ & $2041(17.7)$ & $113239(18.8)$ & 0.03 & 19.4 & 18.8 & 0.02 \\
\hline 5 & $136946(22.3)$ & $2827(24.5)$ & $134119(22.2)$ & 0.05 & 24.7 & 22.2 & 0.06 \\
\hline \multicolumn{8}{|l|}{ Material deprivation } \\
\hline 1 & $119103(19.4)$ & 2795 (24.3) & 116308 (19.3) & 0.12 & 20.0 & 19.2 & 0.02 \\
\hline 2 & 120208 (19.5) & $2380(20.7)$ & $117828(19.5)$ & 0.03 & 20.4 & 19.5 & 0.02 \\
\hline 3 & $117009(19.0)$ & 2097 (18.2) & $114912(19.0)$ & 0.02 & 18.6 & 19.0 & 0.01 \\
\hline 4 & $117529(19.1)$ & $2121(18.4)$ & $115408(19.1)$ & 0.02 & 18.8 & 19.1 & 0.01 \\
\hline 5 & $141364(23.0)$ & $2126(18.5)$ & $139238(23.1)$ & 0.11 & 22.3 & 23.1 & 0.02 \\
\hline \multicolumn{8}{|l|}{ Dependency } \\
\hline 1 & 209834 (34.1) & 3519 (30.5) & 206315 (34.2) & 0.08 & 30.4 & 34.1 & 0.08 \\
\hline 2 & $129753(21.1)$ & 2447 (21.2) & $127306(21.1)$ & 0.00 & 21.7 & 21.1 & 0.01 \\
\hline 3 & 104245 (16.9) & $2045(17.8)$ & 102200 (16.9) & 0.02 & 18.3 & 16.9 & 0.03 \\
\hline 4 & $92197(15.0)$ & $1943(16.9)$ & $90254(15.0)$ & 0.05 & 16.2 & 15.0 & 0.03 \\
\hline 5 & 79184 (12.9) & 1565 (13.6) & 77619 (12.9) & 0.02 & 13.4 & 12.9 & 0.02 \\
\hline \multicolumn{8}{|l|}{ Ethnic concentration } \\
\hline 1 & 81765 (13.3) & $1379(12.0)$ & 80386 (13.3) & 0.04 & 13.4 & 13.3 & 0.00 \\
\hline 2 & $92146(15.0)$ & $1652(14.3)$ & $90494(15.0)$ & 0.02 & 15.2 & 15.0 & 0.00 \\
\hline 3 & $104071(16.9)$ & $1942(16.9)$ & $102129(16.9)$ & 0.00 & 18.0 & 16.9 & 0.03 \\
\hline 4 & $129709(21.1)$ & $2428(21.1)$ & $127281(21.1)$ & 0.00 & 21.5 & 21.1 & 0.01 \\
\hline 5 & $207522(33.7)$ & $4118(35.7)$ & 203404 (33.7) & 0.04 & 31.9 & 33.6 & 0.04 \\
\hline \multicolumn{8}{|l|}{ Prenatal care index†† } \\
\hline Intensive & $12518(2.0)$ & $280(2.4)$ & $12238(2.0)$ & 0.03 & 1.90 & 2.0 & 0.01 \\
\hline Adequate & $167489(27.2)$ & $4468(38.8)$ & $163021(27.0)$ & 0.25 & 25.5 & 26.8 & 0.03 \\
\hline Intermediate & $308121(50.1)$ & $5683(49.3)$ & $302438(50.1)$ & 0.02 & 47.8 & 50.1 & 0.05 \\
\hline Inadequate & $87112(14.2)$ & $894(7.8)$ & $86218(14.3)$ & 0.21 & 16.3 & 14.4 & 0.05 \\
\hline No care/missingł‡ & $39973(6.5)$ & $194(1.7)$ & $39779(6.6)$ & 0.25 & 8.5 & 6.7 & 0.07 \\
\hline \multicolumn{8}{|c|}{ 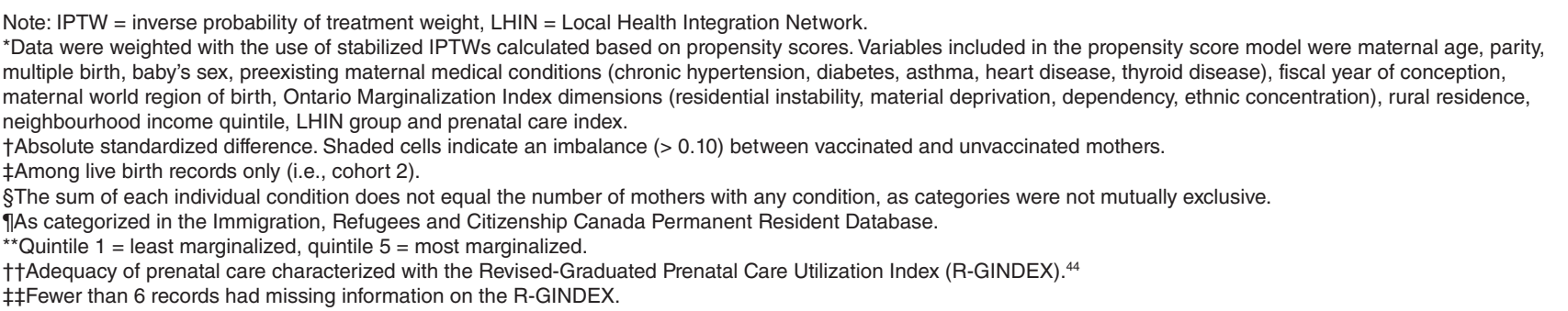 } \\
\hline
\end{tabular}




\begin{tabular}{|c|c|c|c|c|}
\hline \multirow[b]{2}{*}{ Outcome } & \multicolumn{2}{|c|}{ No. (\%) of maternal-infant pairs } & \multirow{2}{*}{$\begin{array}{c}\text { Crude estimate, } \\
\text { RR or HR }(95 \% \mathrm{Cl})\end{array}$} & \multirow{2}{*}{$\begin{array}{c}\text { IPTW-adjusted } \\
\text { estimate, }{ }^{*} \\
\text { RR or HR }(95 \% \mathrm{CI}\end{array}$} \\
\hline & Vaccinated & Unvaccinated & & \\
\hline Cohort 1 (live birth + stillbirth records) & $n=11519$ & $n=603694$ & & \\
\hline Chorioamnionitis $†$ & $139(1.2)$ & $6081(1.0)$ & $1.20(1.01-1.42)$ & $1.17(0.99-1.39)$ \\
\hline Gestational hypertension† & $388(3.4)$ & $23087(3.8)$ & $0.88(0.80-0.97)$ & $0.87(0.78-0.96)$ \\
\hline Postpartum hemorrhage $†$ & $324(2.8)$ & $16916(2.8)$ & $1.00(0.90-1.12)$ & $1.01(0.91-1.13)$ \\
\hline Severe postpartum hemorrhage†‡ & $31(0.3)$ & $2002(0.3)$ & $0.81(0.57-1.16)$ & $0.79(0.55-1.13)$ \\
\hline Preterm birth (<37 wk)§ף & $670(5.8)$ & $44929(7.4)$ & $0.99(0.92-1.07)$ & $0.98(0.91-1.06)$ \\
\hline Very preterm birth (<32 wk)§ף & $66(0.6)$ & $7945(1.3)$ & $1.09(0.85-1.39)$ & $1.10(0.86-1.41)$ \\
\hline Stillbirth§ & $36(0.3)$ & $3249(0.5)$ & $0.99(0.71-1.38)$ & $1.15(0.82-1.60)$ \\
\hline Cohort 2 (live birth records only) & $n=11483$ & $n=600445$ & & \\
\hline Small for gestational age $†$ & $1053(9.2)$ & $57049(9.5)$ & $0.97(0.91-1.02)$ & $0.96(0.90-1.02)$ \\
\hline Neonatal intensive care unit stay $>24 \mathrm{~h} \dagger$ & $713(6.2)$ & $46271(7.7)$ & $0.81(0.75-0.87)$ & $0.82(0.76-0.88)$ \\
\hline Composite neonatal morbidity $\dagger^{\star *}$ & $642(5.6)$ & $42029(7.0)$ & $0.80(0.74-0.86)$ & $0.81(0.75-0.87)$ \\
\hline \multicolumn{5}{|c|}{$\begin{array}{l}\text { Note: } \mathrm{Cl}=\text { confidence interval, } \mathrm{HR}=\text { hazard ratio, IPTW = inverse probability of treatment weight, RR = risk ratio. } \\
\text { *Stabilized IPTWs were used to adjust estimates. } \\
\text { †Estimate values represent RRs that were computed with a log-binomial regression model. } \\
\text { †Postpartum hemorrhage in conjunction with a procedure code for hysterectomy, blood transfusion or other procedure to control bleeding. } \\
\S \text { Estimate values represent HRs that were computed with a Cox model, where tetanus-diphtheria-acellular pertussis (Tdap) vaccination was modelled as a } \\
\text { time-varying exposure. } \\
\text { १Mothers who received Tdap vaccine after } 36 \text { weeks' gestation or } 31 \text { weeks' gestation were treated as unvaccinated in the analyses of preterm birth and very } \\
\text { preterm birth, respectively. } \\
\text { **Adaptation }{ }^{39} \text { of a composite indicator for neonatal morbidity } \\
\text { birth (codes provided in Appendix } 1 \text {, Supplemental Tables } \mathrm{S} 2 \text { and } \mathrm{S} 3 \text { ). }\end{array}$} \\
\hline
\end{tabular}

\section{Sensitivity analyses}

Inclusion of possible Tdap vaccination in our sensitivity analysis identified 2792 additional records, bringing the number of exposed subjects to 14311 . Repeating our analyses with this expanded definition did not alter interpretation of our findings except for chorioamnionitis, which was statistically significant despite the point estimate's being of similar magnitude (Appendix 1, Supplemental Table S5). Adjustment for maternal use of health care services before the index pregnancy had little impact (Appendix 1, Supplemental Table S6).

Sensitivity analyses to assess the potential impact of residual confounding indicated that a harmful unmeasured confounder would have biased estimates downward, particularly if the prevalence were lower in Tdap-vaccinated mothers. For instance, if $20 \%$ of unvaccinated mothers and $10 \%$ of Tdapvaccinated mothers had a risk factor that increased the outcome twofold, we would have underestimated the HRs and RRs of study outcomes by $8 \%$ (Appendix 1, section on bias analyses and Supplemental Tables S7-S16).

\section{Interpretation}

In this large population-based cohort study examining obstetric and perinatal outcomes among more than 11000 Ontario women who received Tdap vaccination during pregnancy, we found no association between vaccination and chorioamnion- itis, postpartum hemorrhage, severe postpartum hemorrhage, preterm birth, very preterm birth, being small for gestational age or stillbirth. We observed reductions in risk for gestational hypertension, a neonatal intensive care unit stay longer than 24 hours and composite neonatal morbidity among Tdap-vaccinated mothers.

Our results concur with evidence that Tdap vaccination during pregnancy is not associated with poor perinatal outcomes, such as preterm birth, $17,19,21,22,25,27,28,48,49$ being small for gestational age $e^{19,22,27,48}$ and stillbirth. ${ }^{17,19,21,23,31-33}$ Although the reduced risks of a neonatal intensive care unit stay exceeding 24 hours and neonatal morbidity may have been due to nonspecific beneficial effects of maternal Tdap vaccination (i.e., vaccine protection beyond the targeted disease ${ }^{50}$ ), limited evidence supports this in the context of maternal vaccination. Given known biases in observational studies on vaccination, ${ }^{51}$ we cannot discount the possibility that residual confounding induced a healthy vaccinee bias.

Individual neonatal morbidities have been examined in several maternal Tdap vaccination studies. A large cohort study with more than 1 million pregnant women (148 817 Tdapvaccinated) showed no increased risks of adverse newborn outcomes, such as respiratory distress and seizures, and a reduced risk of neonatal sepsis among infants born to Tdapvaccinated women. ${ }^{30}$ DeSilva and colleagues ${ }^{18}$ observed no associations between Tdap vaccination during pregnancy and transient tachypnea, neonatal sepsis, neonatal pneumonia, 
respiratory distress syndrome or newborn convulsions. A smaller single-centre study showed similar risks of neonatal complications, including requirement for ventilation, sepsis and intraventricular hemorrhage, between Tdap-vaccineexposed infants and unexposed infants. ${ }^{19} \mathrm{~A}$ New Zealand study of about 70000 infants showed a reduction in respiratory distress syndrome (adjusted odds ratio $0.65,95 \%$ CI 0.52-0.81) among Tdap-vaccine-exposed infants compared to unexposed infants. ${ }^{22}$

Evidence on Tdap vaccination during pregnancy and chorioamnionitis is inconsistent. Three studies showed a small increased risk of chorioamnionitis among Tdap-vaccinated women; none showed increased risks for adverse infant outcomes typically associated with chorioamnionitis, such as preterm birth. ${ }^{18,27,30}$ Like Morgan and colleagues ${ }^{19}$ and Berenson and colleagues, ${ }^{17}$ we found no association with chorioamnionitis or preterm birth. Although the magnitude of our estimate for chorioamnionitis changed minimally in sensitivity analyses, the $95 \%$ CI excluded the null value in 1 such analysis; therefore, it is prudent to continue to monitor this outcome. Our finding that maternal Tdap vaccination was not associated with postpartum hemorrhage aligns with 2 previous studies. ${ }^{23,25}$

We are unaware of any biologic mechanism to explain the protective relation observed between Tdap vaccination and gestational hypertension. Previous studies ${ }^{25,27}$ have not shown an association between Tdap vaccination and this outcome. Residual confounding may partly account for this finding: compared to unvaccinated mothers, those vaccinated may represent a healthier population who engage in behaviours that lower their risk for gestational hypertension. However, our assessment of residual confounding (Appendix 1, section on bias analyses) indicated that even a strong unmeasured protective confounder that was more prevalent in the Tdapvaccinated group is unlikely to have biased the estimate more than $5 \%$. The lack of information about the timing of disease onset precluded accounting for temporality of the association in our analyses; this may have produced an apparent risk reduction. ${ }^{51,52}$ In contrast, Griffin and colleagues, ${ }^{25}$ who were able to account for timing of diagnosis in relation to vaccination, found no association between Tdap vaccination and gestational hypertension.

\section{Limitations}

Strengths of our study include its population-based design, comprising a source population of more than 600000 births. We were able to include perinatal outcomes that are recommended for assessment in maternal vaccination studies, ${ }^{38}$ including 3285 stillbirths (36 exposed); previous stillbirth assessments have been based on very small numbers of events. ${ }^{17,19,21,23,31-33}$

Despite achieving a good balance of baseline covariates in our weighted sample, propensity scores were limited to variables available in the study databases. Our data on maternal smoking, alcohol and drug use, and body mass index likely captured only the most severe forms of these behaviours or characteristics; thus, we cannot dismiss residual confounding by these or other factors. A common unmeasured confounder that is twice as prevalent in unvaccinated women (i.e., $20 \%$ in unvaccinated and $10 \%$ in Tdap-vaccinated) and associated with a twofold increased risk of the outcome could have biased our estimates downward by $8 \%$, potentially obscuring an increased risk. Although data on timing of vaccination were available, we lacked information on disease onset for gestational hypertension and could not determine temporality of this relation. Owing to difficulties of diagnosing chorioamnionitis clinically, outcome misclassification is possible; however, we found no association between maternal Tdap vaccination and sequelae of chorioamnionitis (e.g., preterm birth ${ }^{53}$ ). Future validation studies of this outcome would be valuable.

Ontario currently limits public funding for adult booster Tdap vaccinations to 1 dose. ${ }^{54}$ If care providers refrained from billing for Tdap vaccination to avoid additional costs for the mother, exposure may have been misclassified. Furthermore, vaccine-specific codes were introduced only 7 months before our study period, and incorrect use of new billing codes may have introduced exposure misclassification. ${ }^{46}$ However, results from sensitivity analyses that included possible Tdap vaccinations billed under general vaccination codes were essentially identical to our main analyses. Although the sensitivity of the Tdap vaccination code in our study was unclear, specificity was likely high: validation studies of Ontario vaccination fee codes showed high specificity (about 81\%-96\%) and positive predictive values (about $88 \%-99 \%) .{ }^{46,55}$ With high specificity and low exposure prevalence in our study, any nondifferential exposure misclassification owing to less than perfect sensitivity would have had only a small impact. ${ }^{56}$

\section{Conclusion}

Our findings corroborate existing literature and support the safety of Tdap vaccination during pregnancy. We found no increased risk of postpartum hemorrhage or chorioamnionitis after Tdap vaccination in Ontario; earlier studies from other countries gave equivocal results for these outcomes. This study provides safety information on maternal Tdap vaccination in Canada, which is important for establishing baseline data to inform maternal vaccination practices and providing reassurance when discussing $T$ dap vaccination recommendations with pregnant women. Future studies using data that extend into the period after adoption of Tdap vaccination policy will be important for further developing the evidence base in Canada.

\section{References}

1. Smith T, Rotondo J, Desai S, et al. Pertussis surveillance in Canada: trends to 2012. Can Commun Dis Rep 2014:40:21-30.

2. Jackson DW, Rohani P. Perplexities of pertussis: recent global epidemiological trends and their potential causes. Epidemiol Infect 2014;142:672-84.

3. Tan T, Dalby T, Forsyth K, et al. Pertussis across the globe: recent epidemiologic trends from 2000 to 2013. Pediatr Infect Dis $72015 ; 34: \mathrm{e} 222-32$

4. Chiappini E, Stival A, Galli L, et al. Pertussis re-emergence in the postvaccination era. BMC Infect Dis 2013;13:151.

5. Celentano LP, Massari M, Paramatti D, et al.; EUVAC-NET Group. Resurgence of pertussis in Europe. Pediatr Infect Dis 7 2005;24:761-5.

6. van Hoek AJ, Campbell H, Amirthalingam G, et al. The number of deaths among infants under one year of age in England with pertussis: results of a capture/recapture analysis for the period 2001 to 2011. Euro Surveill 2013;18: 20414. 
7. von König CHW, Halperin S, Riffelmann M, et al. Pertussis of adults and infants. Lancet Infect Dis 2002;2:744-50.

8. Burns DL, Meade BD, Messionnier NE. Pertussis resurgence: perspectives from the Working Group Meeting on pertussis on the causes, possible paths forward, and gaps in our knowledge. 7 Infect Dis 2014;209(Suppl 1):S32-5.

9. Amirthalingam G. Strategies to control pertussis in infants. Arch Dis Child 2013;98:552-5.

10. Kara EO, Campbell H, Ribeiro S, et al. Survey of household contacts of infants with laboratory-confirmed pertussis infection during a national pertussis outbreak in England and Wales. Pediatr Infect Dis 7 2017;36:140-5.

11. Winter K, Glaser C, Watt J, et al.; Centers for Disease Control and Prevention. Pertussis epidemic - California, 2014. MMWR Morb Mortal Wkly Rep 2014;63:1129-32

12. Centers for Disease Control and Prevention. Updated recommendations for use of tetanus toxoid, reduced diphtheria toxoid, and acellular pertussis vaccine (Tdap) in pregnant women - Advisory Committee on Immunization Practices (ACIP), 2012. MMWR Morb Mortal Wkly Rep 2013;62:131-5.

13. Amirthalingam G, Letley L, Campbell H, et al. Lessons learnt from the implementation of maternal immunization programs in England. Hum Vaccin Immunother 2016;12:2934-9.

14. An Advisory Committee Statement (ACS) National Advisory Committee on Immunization (NACI): update on immunization in pregnancy with tetanus toxoid, reduced diphtheria toxoid and reduced acellular pertussis (Tdap) vaccine. Ottawa: Public Health Agency of Canada; 2018; modified 2019 Oct. 9. Cat no HP40-207/2018d-PDF. Available: www.canada.ca/en/public-health/services/ publications/healthy-living/update-immunization-pregnancy-tdap-vaccine.html (accessed 2020 Nov. 17).

15. An Advisory Committee Statement (ACS) National Advisory Committee on Immunization (NACI): update on pertussis vaccination in pregnancy. Ottawa: Public Health Agency of Canada; 2014. Cat no HP40-93/2014E-PDF. Available: https://www.canada.ca/en/public-health/services/publications/healthy -living/update-pertussis-vaccination-pregnancy.html (accessed 2020 Nov. 17).

16. Gruslin A, Steben M, Halperin S, et al. Immunization in pregnancy. 7 Obstet Gynaecol Can 2008:30:1149-54.

17. Berenson AB, Hirth JM, Rahman M, et al. Maternal and infant outcomes among women vaccinated against pertussis during pregnancy. Hum Vaccin Immunother 2016;12:1965-71.

18. DeSilva M, Vazquez-Benitez G, Nordin JD, et al. Maternal Tdap vaccination and risk of infant morbidity. Vaccine 2017;35:3655-60.

19. Morgan JL, Baggari SR, McIntire DD, et al. Pregnancy outcomes after antepartum tetanus, diphtheria, and acellular pertussis vaccination. Obstet Gynecol 2015;125:1433-8.

20. Munoz FM, Bond NH, Maccato M, et al. Safety and immunogenicity of tetanus diphtheria and acellular pertussis (Tdap) immunization during pregnancy in mothers and infants: a randomized clinical trial. FAMA 2014;311:1760-9.

21. Shakib JH, Korgenski K, Sheng X, et al. Tetanus, diphtheria, acellular pertussis vaccine during pregnancy: pregnancy and infant health outcomes. 7 Pediatr 2013;163:1422-6.e1-4.

22. Petousis-Harris H, Jiang Y, Yu L, et al. A retrospective cohort study of safety outcomes in New Zealand infants exposed to Tdap vaccine in utero. Vaccines (Basel) 2019;7:147.

23. Donegan K, King B, Bryan P. Safety of pertussis vaccination in pregnant women in UK: observational study. BM7 2014;349:g4219.

24. Fortner KB, Edwards KM, Broder KR, et al. Reactogenicity of tetanus toxoid, reduced diphtheria toxoid, and acellular pertussis vaccine (Tdap) in pregnant women. Am 7 Obstet Gynecol 2016;214(Suppl):S193-4.

25. Griffin JB, Yu L, Watson D, et al. Pertussis Immunisation in Pregnancy Safety (PIPS) study: a retrospective cohort study of safety outcomes in pregnant women vaccinated with Tdap vaccine. Vaccine 2018;36:5173-9.

26. Petousis-Harris H, Walls $\mathrm{T}$, Watson $\mathrm{D}$, et al. Safety of Tdap vaccine in pregnant women: an observational study. BM7 Open 2016;6:e010911.

27. Kharbanda EO, Vazquez-Benitez G, Lipkind HS, et al. Evaluation of the association of maternal pertussis vaccination with obstetric events and birth outcomes. 7AMA 2014:312:1897-904.

28. Sukumaran L, McCarthy NL, Kharbanda EO, et al. Association of Tdap vaccination with acute events and adverse birth outcomes among pregnant women with prior tetanus-containing immunizations. 7AMA 2015;314:1581-7.

29. Kharbanda EO, Vazquez-Benitez G, Lipkind HS, et al. Maternal Tdap vaccination: coverage and acute safety outcomes in the vaccine safety datalink, 2007-2013. Vaccine 2016;34:968-73.

30. Layton JB, Butler AM, Li D, et al. Prenatal Tdap immunization and risk of maternal and newborn adverse events. Vaccine 2017;35:4072-8.

31. Hoang HTT, Leuridan E, Maertens $\mathrm{K}$, et al. Pertussis vaccination during pregnancy in Vietnam: results of a randomized controlled trial Pertussis vaccination during pregnancy. Vaccine 2016;34:151-9.

32. Sancovski M, Mesaros N, Feng Y, et al. Safety of reduced antigen content diphtheria-tetanus-acellular pertussis vaccine when administered during pregnancy as part of the maternal immunization program in Brazil: a single center, observational, retrospective, cohort study. Hum Vacin Immunother 2019;15:2873-81.

33. Vygen-Bonnet S, Hellenbrand W, Garbe E, et al. Safety and effectiveness of acellular pertussis vaccination during pregnancy: a systematic review. $B M C$ Infect Dis 2020;20:136.
34. ICES data dictionary. MOMBABY. Toronto: ICES. Available: https:// datadictionary.ices.on.ca/Applications/DataDictionary/Library.aspx?Library= MOMBABY (accessed 2020 Nov. 17).

35. Talge NM, Mudd LM, Sikorskii A, et al. United States birth weight reference corrected for implausible gestational age estimates. Pediatrics 2014;133:844-53.

36. Basso O, Wilcox AJ. Might rare factors account for most of the mortality of preterm babies? Epidemiology 2011;22:320-7

37. Kramer MS, Platt RW, Wen SW, et al.; Fetal/Infant Health Study Group of the Canadian Perinatal Surveillance System. A new and improved populationbased Canadian reference for birth weight for gestational age. Pediatrics 2001; 108:E35.

38. Bonhoeffer J, Kochhar S, Hirschfeld S, et al.; GAIA project participants. Global alignment of immunization safety assessment in pregnancy - the GAIA project. Vaccine 2016;34:5993-7.

39. Ramage K, Grabowska K, Silversides C, et al. Association of adult congenital heart disease with pregnancy, maternal, and neonatal outcomes. $7 A M A$ Netw Open 2019;2:e193667.

40. Lain SJ, Algert CS, Nassar N, et al. Incidence of severe adverse neonatal outcomes: use of a composite indicator in a population cohort. Matern Child Health 7 2012;16:600-8.

41. Yang D, Dalton JE. A unified approach to measuring the effect size between two groups using SAS®. SAS Global Forum; 2012. Available: https://support.sas. com/resources/papers/proceedings12/335-2012.pdf (accessed 2020 Nov. 17).

42. Austin PC. An introduction to propensity score methods for reducing the effects of confounding in observational studies. Multivariate Behav Res 2011;46:399-424.

43. Kharbanda EO, Vazquez-Benitez G, Lipkind H, et al. Receipt of pertussis vaccine during pregnancy across 7 Vaccine Safety Datalink sites. Prev Med 2014;67:316-9.

44. Alexander GR, Kotelchuck M. Quantifying the adequacy of prenatal care: a comparison of indices. Public Health Rep 1996;111:408-18, discussion 419.

45. Austin PC, Stuart EA. Moving towards best practice when using inverse probability of treatment weighting (IPTW) using the propensity score to estimate causal treatment effects in observational studies. Stat Med 2015;34:3661-79.

46. Schwartz KL, Tu K, Wing L, et al. Validation of infant immunization billing codes in administrative data. Hum Vaccin Immunother 2015;11:1840-7.

47. Schneeweiss S. Sensitivity analysis and external adjustment for unmeasured confounders in epidemiologic database studies of therapeutics. Pharmacoepidemiol Drug Saf 2006;15:291-303.

48. Sukumaran L, McCarthy NL, Kharbanda EO, et al. Safety of tetanus toxoid, reduced diphtheria toxoid, and acellular pertussis and influenza vaccinations in pregnancy. Obstet Gynecol 2015;126:1069-74.

49. Hall C, Abramovitz LM, Bukowinski AT, et al. Safety of tetanus, diphtheria, and acellular pertussis vaccination among pregnant active duty U.S. military women. Vaccine 2020;38:1982-8.

50. Marchant A, Sadarangani M, Garand M, et al. Maternal immunisation: collaborating with mother nature. Lancet Infect Dis 2017;17:e197-208.

51. Savitz DA, Fell DB, Ortiz JR, et al. Does influenza vaccination improve pregnancy outcome? Methodological issues and research needs. Vaccine 2015;33: 6430-5.

52. Vazquez-Benitez G, Kharbanda EO, Naleway AL, et al. Risk of preterm or small-for-gestational-age birth after influenza vaccination during pregnancy: caveats when conducting retrospective observational studies. Am 7 Epidemiol 2016;184:176-86.

53. Gagliardi L, Rusconi F, Bellù R, et al. Association of maternal hypertension and chorioamnionitis with preterm outcomes. Pediatrics 2014;134:e154-61.

54. Publicly funded immunization schedules for Ontario - August 2011. Toronto: Polyclinic Family and Specialty Medicine; 2014. Available: http://polyclinic. $\mathrm{ca} / 2 / \mathrm{wp}$-content/uploads/2014/08/Publicly-Funded-Immunization-Schedules -for-Ontario.pdf (accessed 2020 Nov. 17).

55. Schwartz KL, Jembere N, Campitelli MA, et al. Using physician billing claims from the Ontario Health Insurance Plan to determine individual influenza vaccination status: an updated validation study. CMA7 Open 2016;4:E463-70.

56. Szklo M, Nieto FJ. Epidemiology: beyond the basics. 2nd ed. Burlington (MA): Jones and Bartlett Publishers; 2007:109-50.

Affiliations: Children's Hospital of Eastern Ontario Research Institute (Fakhraei, Sucha, Fell); Ottawa Hospital Research Institute (Fakhraei, Hawken, Wilson, Walker), Ottawa, Ont.; ICES Central (Crowcroft, Hawken, Kwong, Fell); University of Toronto (Crowcroft, Bolotin, Biringer, Dubey, Jamieson, Kwong); Public Health Ontario (Bolotin, Jamieson, Kwong), Toronto, Ont.; ICES uOttawa (Sucha); University of Ottawa (Hawken, Cook, Walker, Laverty, Fell); Bruyère Research Institute (Wilson), Ottawa, Ont.; Kingston Health Sciences Centre (Gaudet); Queen's University (Gaudet), Kingston, Ont.; Public Health England (Amirthalingam), London, UK; Mount Sinai Hospital (Biringer), Toronto, Ont.; Society of Obstetricians and Gynaecologists of Canada (Cook), Ottawa, Ont.; Toronto Public Health (Dubey), Toronto, Ont.; Canadian Centre for Vaccinology (Halperin), Dalhousie University and IWK Health Centre, Halifax, NS; Vaccine Evaluation Center (Sadarangani), BC Children's Hospital Research Institute; Department of Pediatrics (Sadarangani), University of British Columbia, Vancouver, BC 
Contributors: Deshayne Fell, Natasha Crowcroft and Shelly Bolotin conceived the study and designed the initial study protocol and analytic approach. Romina Fakhraei, Meghan Laverty, Jeff Kwong and Ewa Sucha made further contributions to the study design and analytic approach. Romina Fakhraei, Meghan Laverty and Ewa Sucha linked the administrative data sources to create the study cohort and performed the statistical analyses, which were supervised by Deshayne Fell. Romina Fakhraei drafted the manuscript. All of the authors contributed to data interpretation, revised the manuscript for important intellectual content, approved the final version to be published and agreed to be accountable for all aspects of the work.

Funding: This study was supported by operating grants PJT-159519 and MY7-161351 from the Canadian Institutes of Health Research and by ICES, which is funded by an annual grant from the Ontario Ministry of Health.

Content licence: This is an Open Access article distributed in accordance with the terms of the Creative Commons Attribution (CC BY-NCND 4.0) licence, which permits use, distribution and reproduction in any medium, provided that the original publication is properly cited, the use is noncommercial (i.e., research or educational use), and no modifications or adaptations are made. See: https://creativecommons.org/licenses/by-nc $-\mathrm{nd} / 4.0 \%$
Data sharing: The data set from this study is held securely in coded form at ICES. Although data-sharing agreements prohibit ICES from making the data set publicly available, access may be granted to those who meet prespecified criteria for confidential access, available at https://www.ices. on.ca/DAS. The full data set creation plan and underlying analytic code are available from the authors on request, with the understanding that the computer programs may rely on coding templates or macros that are unique to ICES and, therefore, are inaccessible or may need modification.

Acknowledgement: The authors thank Immigration, Refugees and Citizenship Canada for providing data to ICES, where the analyses were conducted.

Disclaimer: Parts of this material are based on data and information compiled and provided by the Ontario Ministry of Health, the Canadian Institute for Health Information, and Immigration, Refugees and Citizenship Canada. The analyses, conclusions, opinions and statements expressed herein are solely those of the authors and do not reflect those of the funding or data sources; no endorsement is intended or should be inferred.

Supplemental information: For reviewer comments and the original submission of this manuscript, please see www.cmajopen.ca/content/9/2/ E349/suppl/DC1. 